\title{
COMPATIBILITY SUPPORT MAPPINGS IN EFFECT ALGEBRAS
}

\author{
GEJZA JENČA
}

\begin{abstract}
We give a characterization of subsets of effect algebras, that can be embedded into a range of an observable. To give this characterization, we introduce a new notion of compatibility support mappings.
\end{abstract}

\section{INTRODUCTION AND MOTIVATION}

Question 1. Let $S$ be a set of effects on a separable Hilbert space $\mathbb{H}$. Is there a measurable space $(X, \mathcal{A})$ and a POV-measure $\alpha:(X, \mathcal{A}) \rightarrow \mathcal{E}(\mathbb{H})$ such that $S$ is a subset of the range of $\alpha$ ?

If $S$ consists only of orthogonal projections (that means, idempotent effects), then the answer is simple: $S$ is a subset of the range of a POV-measure iff the elements of $S$ commute. On the other hand, if there are non-idempotent effects in $S$, the answer is not known.

In the present paper, we examine a related question:

Question 2. If $S$ is a subset of an effect algebra $E$, is there a Boolean algebra $B$ and a morphism of effect algebras $\alpha: B \rightarrow E$ such that $S \subseteq \alpha(B)$ ?

This can be considered as a quantum-logical version of Question 1. We prove that, given subset $S$ of an effect algebra $E$ such that $1 \in S$, there exist a Boolean algebra $B$ and a morphism $\alpha: B \rightarrow E$ with $S \subseteq \alpha(B)$ if and only if there is a mapping $\llbracket ., . \rrbracket: \operatorname{Fin}(S) \times \operatorname{Fin}(S) \rightarrow E$ satisfying certain properties. We call them compatibility support mappings. The proof uses a modification of the limit techniques introduced in [3].

We show that compatibility support mappings, and hence pairs $(B, \alpha)$, exist whenever $S$ is an MV-algebra or $S$ is a pairwise commuting set of effects on a Hilbert space. We prove several properties of strong compatibility support maps, generalizing the properties of the prototype Example 2.

The results presented in this paper are more general than the results from an earlier paper [7, where only interval effect algebras were considered. In that paper, a related notion of witness 06 was introduced to characterize coexistent subsets of interval effect algebras.

In the last section, we examine connections between compatibility support mappings and witness mappings. We prove that, for a subset $S$ of an interval effect algebra, every compatibility support map for $S$ gives rise to a witness mapping for $S$. We do not know whether this relationship is a one-to-one correspondence.

1991 Mathematics Subject Classification. Primary: 03G12, Secondary: 06F20, 81P10.

Key words and phrases. effect algebra, observables.

This research is supported by grants VEGA G-1/0080/10 of MŠ SR, Slovakia and by the Slovak Research and Development Agency under the contracts No. APVT-51-032002, APVV-0071-06. 


\section{DEFINITIONS AND BASIC RELATIONSHIPS}

An effect algebra is a partial algebra $(E ; \oplus, 0,1)$ with a binary partial operation $\oplus$ and two nullary operations 0,1 satisfying the following conditions.

(E1) If $a \oplus b$ is defined, then $b \oplus a$ is defined and $a \oplus b=b \oplus a$.

(E2) If $a \oplus b$ and $(a \oplus b) \oplus c$ are defined, then $b \oplus c$ and $a \oplus(b \oplus c)$ are defined and $(a \oplus b) \oplus c=a \oplus(b \oplus c)$.

(E3) For every $a \in E$ there is a unique $a^{\prime} \in E$ such that $a \oplus a^{\prime}=1$.

(E4) If $a \oplus 1$ exists, then $a=0$

Effect algebras were introduced by Foulis and Bennett in their paper [5]. Independently, Kôpka and Chovanec introduced an essentially equivalent structure called D-poset (see [8]). Another equivalent structure, called weak orthoalgebras was introduced by Giuntini and Greuling in 6 .

For brevity, we denote the effect algebra $(E, \oplus, 0,1)$ by $E$. In an effect algebra $E$, we write $a \leq b$ iff there is $c \in E$ such that $a \oplus c=b$. It is easy to check that every effect algebra is cancellative, thus $\leq$ is a partial order on $E$. In this partial order, 0 is the least and 1 is the greatest element of $E$. Moreover, it is possible to introduce a new partial operation $\ominus ; b \ominus a$ is defined iff $a \leq b$ and then $a \oplus(b \ominus a)=b$. It can be proved that $a \oplus b$ is defined iff $a \leq b^{\prime}$ iff $b \leq a^{\prime}$. It is usual to denote the domain of $\oplus$ by $\perp$. If $a \perp b$, we say that $a$ and $b$ are orthogonal.

Example 1. The prototype example of an effect algebra is the standard effect algebra $\mathcal{E}(\mathbb{H})$. Let $\mathbb{H}$ be a Hilbert space. Let $\mathcal{S}(\mathbb{H})$ be the set of all bounded self-adjoint operators. on $\mathbb{H}$. Let $\mathbb{I}$ be the identity operator $\mathbb{H}$.

For $A, B \in \mathcal{S}(\mathbb{H})$, write $A \leq B$ if and only if, for all $x \in \mathbb{H},\langle A x, x\rangle \leq\langle B x, x\rangle$.

Put $\mathcal{E}(\mathbb{H})=\{X \in \mathcal{S}(\mathbb{H}): 0 \leq X \leq I\}$ and for $A, B \in \mathcal{E}(\mathbb{H})$ define $A \oplus B$ iff $A \oplus B \leq I, A \oplus B=A+B$. Then $(\mathcal{E}(\mathbb{H}), \oplus, 0, I)$ is an effect algebra. The elements of $\mathcal{E}(\mathbb{H})$ are called Hilbert space effects.

An effect algebra $E$ is lattice ordered iff $(E, \leq)$ is a lattice. An effect algebra is an orthoalgebra iff $a \perp a$ implies $a=0$. An orthoalgebra that is lattice ordered is an orthomodular lattice.

An $M V$-effect algebra is a lattice ordered effect algebra $M$ in which, for all $a, b \in$ $M,(a \vee b) \ominus a=b \ominus(a \wedge b)$. It is proved in 4 that there is a natural, one-to one correspondence between MV-effect algebras and MV-algebras given by the following rules. Let $(M, \oplus, 0,1)$ be an MV-effect algebra. Let $\boxplus$ be a total operation given by $x \boxplus y=x \oplus\left(x^{\prime} \wedge y\right)$. Then $\left(M, \boxplus,^{\prime}, 0\right)$ is an MV-algebra. Similarly, let $(M, \boxplus, \neg, 0)$ be an MV-algebra. Restrict the operation $\boxplus$ to the pairs $(x, y)$ satisfying $x \leq y^{\prime}$ and call the new partial operation $\oplus$. Then $(M, \oplus, 0, \neg 0)$ is an MV-effect algebra.

Among lattice ordered effect algebras, MV-effect algebras can be characterized in a variety of ways. Three of them are given in the following proposition.

Proposition 1. 1, 4] Let E be a lattice ordered effect algebra. The following are equivalent

(a) $E$ is an $M V$-effect algebra.

(b) For all $a, b \in E, a \wedge b=0$ implies $a \leq b^{\prime}$.

(c) For all $a, b \in E, a \ominus(a \wedge b) \leq b^{\prime}$.

(d) For all $a, b \in E$, there exist $a_{1}, b_{1}, c \in E$ such that $a_{1} \oplus b_{1} \oplus c$ exists, $a_{1} \oplus c=a$ and $b_{1} \oplus c=b$. 
Let $B$ be a Boolean algebra and let $E$ be an effect algebra. An observable is a mapping $\alpha: B \rightarrow E$ such that $\alpha(0)=0, \alpha(1)=1$ and for every $x, y \in B$ such that $x \wedge y=0, \phi(x \vee y)=\phi(x) \oplus \phi(y)$.

\section{Compatibility SupPort MAPPINGS — DEFINITION AND EXAMPleS}

In this section we introduce (strong) compatibility support mappings and present two examples.

Definition 1. Let $E$ be an effect algebra, let $S \subseteq E$ be such that $1 \in S$. We say that $\llbracket ., . \rrbracket: F i n(S) \times F i n(S) \rightarrow E$ is a compatibility support mapping for $S$ if and only if the following conditions are satisfied.

(a) If $V_{1} \subseteq V_{2}$, then $\llbracket U, V_{1} \rrbracket \leq \llbracket U, V_{2} \rrbracket$.

(b) $\llbracket U, V \rrbracket \leq \llbracket U,\{1\} \rrbracket$.

(c) $\llbracket U, \emptyset \rrbracket=0$.

(d) $\llbracket \emptyset,\{c\} \rrbracket=c$.

(e) If $c \notin U \cup V$, then $\llbracket U \cup\{c\},\{1\} \rrbracket \ominus \llbracket U \cup\{c\}, V \rrbracket=\llbracket U, V \cup\{c\} \rrbracket \ominus \llbracket U, V \rrbracket$

A compatibility mapping is strong if and only if the following condition is satisfied.

$\left(\mathrm{e}^{*}\right)$ For all $c, \llbracket U \cup\{c\},\{1\} \rrbracket \ominus \llbracket U \cup\{c\}, V \rrbracket=\llbracket U, V \cup\{c\} \rrbracket \ominus \llbracket U, V \rrbracket$

Note that $\left(\mathrm{e}^{*}\right)$ implies (e).

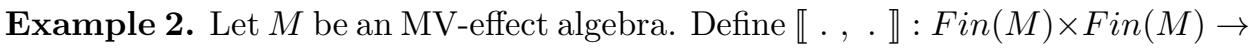
$E$ by

$$
\llbracket U, V \rrbracket=(\bigwedge U) \wedge(\bigvee V)
$$

Then $\llbracket ., . \rrbracket$ is a strong compatibility support mapping. The conditions (a)-(d) are easy to prove. Let us prove $\left(\mathrm{e}^{*}\right)$.

$$
\begin{array}{r}
\llbracket U, V \cup\{c\} \rrbracket \ominus \llbracket U, V \rrbracket=(\bigwedge U) \wedge(c \vee(\bigvee V)) \ominus((\bigwedge U) \wedge(\bigvee V))= \\
=((\bigwedge U) \wedge c) \vee((\bigwedge U) \wedge(\bigvee V)) \ominus((\bigwedge U) \wedge(\bigvee V))= \\
=((\bigwedge U) \wedge c) \ominus((\bigwedge U) \wedge c \wedge(\bigvee V))= \\
=\llbracket U \cup\{c\},\{1\} \rrbracket \ominus \llbracket U \cup\{c\}, V \rrbracket
\end{array}
$$

Example 3. Let $\sqcup$ be an operation on the set of all operators on a Hilbert space $\mathbb{H}$ given by

$$
a \sqcup b:=a+b-a b .
$$

It is easy to check that $\sqcup$ is associative with neutral element 0 .

If $a$ and $b$ are commuting effects, then $a . b$ is an effect with $a . b \leq a, b$. Moreover, $a \sqcup b$ is an effect. Indeed, since $a, b$ are commuting effects, $1-a, 1-b$ are commuting effects. Since $1-a, 1-b$ are commuting effects, $(1-a) \cdot(1-b)$ is an effect and

$$
1-(1-a) \cdot(1-b)=1-(1-a-b+a b)=a+b-a b
$$

is an effect.

Let $S$ be a set of commuting effects with $1 \in S$; there exists a commutative $C^{*}$ algebra $A$ with $S \subseteq A$. The operations $\sqcup$, . are commutative and associative on $A \cap \mathcal{E}(\mathbb{H}) \supseteq S$.

Let $U, V$ be a finite subsets of $S$. Write $\Pi U$ for the product of elements of $U$. Write $\bigsqcup \emptyset=0, \bigsqcup\{c\}=c$ and, for $V=\left\{v_{1}, \ldots, v_{n}\right\}$ with $n>1$, write

$$
\bigsqcup V=v_{1} \sqcup \cdots \sqcup v_{n} .
$$


Define $\llbracket ., . \rrbracket: \operatorname{Fin}(S) \times \operatorname{Fin}(S) \rightarrow E$

$$
\llbracket U, V \rrbracket=\left(\prod U\right) \cdot(\bigsqcup V) .
$$

Let us prove that $\llbracket .$, . \ is a compatibility support mapping.

Proof of condition (a): Suppose that $V_{1} \subseteq V_{2}$. We need to prove that $\llbracket U, V_{1} \rrbracket \leq$ $\llbracket U, V_{2} \rrbracket$. Let us prove that $\bigsqcup V_{1} \leq \bigsqcup V_{2}$. Since $V_{1} \subseteq V_{2}$, we may write

$$
\begin{array}{r}
\bigsqcup V_{2}=\left(\bigsqcup V_{1}\right) \sqcup\left(\bigsqcup\left(V_{2} \backslash V_{1}\right)\right)= \\
=\left(\bigsqcup V_{1}\right)+\left(\bigsqcup\left(V_{2} \backslash V_{1}\right)\right)-\left(\bigsqcup V_{1}\right) \cdot\left(\bigsqcup\left(V_{2} \backslash V_{1}\right)\right)
\end{array}
$$

Therefore,

$$
\left(\bigsqcup V_{2}\right)-\left(\bigsqcup V_{1}\right)=\left(\bigsqcup\left(V_{2} \backslash V_{1}\right)\right)-\left(\bigsqcup V_{1}\right) \cdot\left(\bigsqcup\left(V_{2} \backslash V_{1}\right)\right) \geq 0,
$$

so $\bigsqcup V_{1} \leq \bigsqcup V_{2}$. Since $\bigsqcup V_{1} \leq \bigsqcup V_{2}$,

$$
\llbracket U, V_{1} \rrbracket=\left(\prod U\right) \cdot\left(\bigsqcup V_{1}\right) \leq\left(\prod U\right) \cdot\left(\bigsqcup V_{1}\right)=\llbracket U, V_{2} \rrbracket .
$$

The conditions (b)-(d) are trivially satisfied.

Proof of condition the (e):

$$
\begin{array}{r}
\llbracket U, V \cup\{c\} \rrbracket-\llbracket U, V \rrbracket=\left(\prod U\right) \cdot(c \sqcup \bigsqcup V)-\left(\prod U\right) \cdot(\bigsqcup V)= \\
=\left(\prod U\right) \cdot(c+\bigsqcup V-c \cdot(\bigsqcup V))-\left(\prod U\right) \cdot(\bigsqcup V)= \\
=\left(\prod U\right) \cdot c+\left(\prod U\right) \cdot(\bigsqcup V)-\left(\prod U\right) \cdot c \cdot(\bigsqcup V)-\left(\prod U\right) \cdot(\bigsqcup V)= \\
=\left(\prod U\right) . c-\left(\prod U\right) . c \cdot(\bigsqcup V)=\llbracket U \cup\{c\},\{1\} \rrbracket \ominus \llbracket U \cup\{c\}, V \rrbracket
\end{array}
$$

Note that, if $S$ contains some non-idempotent $c$, then $\llbracket ., . \rrbracket$ is not strong. To see that $\left(\mathrm{e}^{*}\right)$ is not satisfied, put $U=V=\{c\}$ and compute

$$
\begin{array}{r}
\llbracket U \cup\{c\},\{1\} \rrbracket \ominus \llbracket U \cup\{c\}, V \rrbracket=c \ominus c . c \neq 0 \\
\llbracket U, V \cup\{c\} \rrbracket \ominus \llbracket U, V \rrbracket=c . c \ominus c . c=0
\end{array}
$$

\section{ObSERVABles From COMPATIBILITy SUPPORT MAPPINGS}

The aim of this section is to prove that for every $S$ such that $S \cup\{1\}$ admits a compatibility support mapping, then $S$ is coexistent.

The direct limit method used here is a dual of the projective limit method introduced in $[3$. See also 9 for another application of the projective limit method.

Several proofs in this section (Lemma 3 through Theorem 1) are very similar, or even the same, as in [7. The reason for this is that they are basically an application of Lemma 2, which is the Proposition 4 of [7. However, the author decided to include them here, to keep the present paper more streamlined.

Running assumption 1. In this section, we assume the following.

- $E$ is an effect algebra.

- $S$ is a subset of $E$ with $1 \in S$.

- $\llbracket ., . \rrbracket: \operatorname{Fin}(S) \times \operatorname{Fin}(S) \rightarrow S$ is a compatibility support mapping.

Lemma 1. For all $c \in S, \llbracket\{c\},\{1\} \rrbracket=c$. 
Proof. Put $U=V=\emptyset$ in condition (e) of Definition 1, We see that

$$
\llbracket\{c\},\{1\} \rrbracket \ominus \llbracket\{c\}, \emptyset \rrbracket=\llbracket \emptyset,\{c\} \rrbracket \ominus \llbracket \emptyset, \emptyset \rrbracket .
$$

By conditions (c) and (d), this implies that $\llbracket\{c\},\{1\} \rrbracket=c$.

Let us write, for $A, X \in F i n(S)$ such that $X \subseteq A$,

$$
D(X, A)=\llbracket X,\{1\} \rrbracket \ominus \llbracket X, A \backslash X \rrbracket .
$$

Lemma 2. Let $A, X \in F i n(S), X \subseteq A$ and let $c \in S$ be such that $c \notin A$. Then

$$
D(X, A)=D(X, A \cup\{c\}) \oplus D(X \cup\{c\}, A \cup\{c\}) .
$$

Proof. We see that

$$
\begin{gathered}
D(X, A \cup\{c\})=\llbracket X,\{1\} \rrbracket \ominus \llbracket X,\{c\} \cup(A \backslash X) \rrbracket \\
D(X \cup\{c\}, A \cup\{c\})=\llbracket X \cup\{c\},\{1\} \rrbracket \ominus \llbracket X \cup\{c\}, A \backslash X \rrbracket
\end{gathered}
$$

and, by condition (e) of Definition 1, we see that

$$
\llbracket X \cup\{c\},\{1\} \rrbracket \ominus \llbracket X \cup\{c\}, A \backslash X \rrbracket=\llbracket X,\{c\} \cup(A \backslash X) \rrbracket \ominus \llbracket X, A \backslash X \rrbracket .
$$

Therefore,

$$
\begin{array}{r}
D(X, A \cup\{c\}) \oplus D(X \cup\{c\}, A \cup\{c\})= \\
=(\llbracket X,\{1\} \rrbracket \ominus \llbracket X,\{c\} \cup(A \backslash X) \rrbracket) \oplus(\llbracket X,\{c\} \cup(A \backslash X) \rrbracket \ominus \llbracket X, A \backslash X \rrbracket)= \\
=\llbracket X,\{1\} \rrbracket \ominus \llbracket X, A \backslash X \rrbracket=D(X, A) .
\end{array}
$$

Lemma 3. Let $C, A, X \in F i n(S)$ be such that $X \subseteq A$ and $C \cap A=\emptyset$. Then $(D(X \cup Y, A \cup C))_{Y \subseteq C}$ is an orthogonal family and

$$
\bigoplus_{Y \subseteq C} D(X \cup Y, A \cup C)=D(X, A) .
$$

Proof. The proof goes by induction with respect to $|C|$.

For $C=\emptyset$, Lemma 3 is trivially true.

Assume that Lemma 3 holds for all $C$ with $|C|=n$ and let $c \in S, c \notin A \cup C$. Let us consider the family

$$
(D(X \cup Z, A \cup C \cup\{c\}))_{Z \subseteq C \cup\{c\}} .
$$

For every $Z \subseteq C \cup\{c\}$, either $c \in Z$ or $c \notin Z$, so either $Z=Y \cup\{c\}$ or $Z=Y$, for some $Y \subseteq C$. Therefore, we can write

$$
\begin{array}{r}
(D(X \cup Z, A \cup C \cup\{c\}))_{Z \subseteq C \cup\{c\}}= \\
(D(X \cup Y, A \cup C \cup\{c\}), D(X \cup Y \cup\{c\}, A \cup C \cup\{c\}))_{Y \subseteq C .} .
\end{array}
$$

By Lemma 2,

$$
D(X \cup Y, A \cup C \cup\{c\}) \oplus D(X \cup Y \cup\{c\}, A \cup C \cup\{c\})=D(X \cup Y, A \cup C) .
$$

It only remains to apply the induction hypothesis to finish the proof.

Corollary 1. For every $A \in \operatorname{Fin}(S),(D(X, A))_{X \subseteq A}$ is a decomposition of unit. 
Proof. Obviously,

$$
D(\emptyset, \emptyset)=\llbracket \emptyset,\{1\} \rrbracket \ominus \llbracket \emptyset, \emptyset \rrbracket=1 \ominus 0=1 .
$$

By Lemma 3 ,

$$
\bigoplus_{X \subseteq A}(D(\emptyset \cup X, \emptyset \cup A))=D(\emptyset, \emptyset)
$$

Corollary 2. For every $A \in \operatorname{Fin}(S)$, the mapping $\alpha_{A}: 2^{\left(2^{A}\right)} \rightarrow E$ given by

$$
\alpha_{A}(\mathbb{X})=\bigoplus_{X \in \mathbb{X}} D(X, A)
$$

is a simple observable.

Proof. The atoms of $2^{\left(2^{A}\right)}$ are of the form $\{X\}$, where $X \subseteq A$. By Corollary 1 $\left(\alpha_{A}(\{X\}): X \subseteq A\right)$ is a decomposition of unit; the remainder of the proof is trivial.

For $A, B \in \operatorname{Fin}(S)$ with $A \subseteq B$, let us define mappings $g_{B}^{A}: 2^{\left(2^{A}\right)} \rightarrow 2^{\left(2^{B}\right)}$

$$
g_{B}^{A}(\mathbb{X})=\left\{X \cup C_{0}: X \in \mathbb{X} \text { and } C_{0} \subseteq(B \backslash A)\right\}
$$

and let us write $\mathcal{G}$ for the collection of all such mappings.

It is an easy exercise to prove that every $g_{B}^{A} \in \mathcal{G}$ is an injective homomorphism of Boolean algebras and that $\left(\left(2^{\left(2^{A}\right)}: A \in \operatorname{Fin}(S)\right), \mathcal{G}\right)$ is a direct family of Boolean algebras.

Let us prove that the mappings $g_{B}^{A}$ behave well with respect to the observables $\alpha_{A}$ and $\alpha_{B}$.

Lemma 4. Let $A, B \in F i n(S)$ with $A \subseteq B$. The diagram

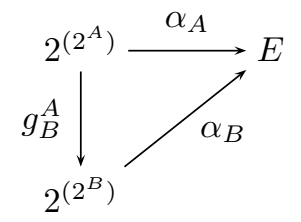

commutes.

Proof. For all $\mathbb{X} \in 2^{\left(2^{A}\right)}$,

$$
\begin{array}{r}
\alpha_{B}\left(g_{B}^{A}(\mathbb{X})\right)=\alpha_{B}\left(\left\{X \cup C_{0}: X \in \mathbb{X} \text { and } C_{0} \subseteq(B \backslash A)\right\}\right)= \\
=\bigoplus\left(D\left(X \cup C_{0}, B\right): X \in \mathbb{X} \text { and } C_{0} \subseteq(B \backslash A)\right)= \\
=\bigoplus_{X \in \mathbb{X}}\left(\bigoplus_{C_{0} \subseteq(B \backslash A)} D\left(X \cup C_{0}, B\right)\right)
\end{array}
$$

Put $Y:=C_{0}, C:=B \backslash A$; by Lemma 3 ,

Therefore,

$$
\bigoplus_{C_{0} \subseteq(B \backslash A)} D\left(X \cup C_{0}, B\right)=D(X, A) .
$$

$$
\alpha_{B}\left(g_{B}^{A}(\mathbb{X})\right)=\bigoplus_{X \in \mathbb{X}} D(X, A)=\alpha_{A}(\mathbb{X})
$$

and the diagram commutes. 
Corollary 3. For every $B \in \operatorname{Fin}(S), B$ is a subset of the range of $\alpha_{B}$.

Proof. We need to prove that every $a \in B$ is an element of the range of $\alpha_{B}$. For $B=\emptyset$, this is trivial.

Suppose that $B$ is nonempty and let $a \in B$. Let $A=\{a\}$. and let $X=$ $g_{B}^{A}(\{\{a\}\})$. By Lemma 4 .

$$
\alpha_{B}(X)=\alpha_{B}\left(g_{B}^{A}(\{\{a\}\})\right)=\alpha_{A}(\{\{a\}\}),
$$

and we see that, by (c) of Definition 1 and by Lemma 1

$\alpha_{A}(\{\{a\}\})=\alpha_{\{a\}}(\{\{a\}\})=D(\{a\},\{a\})=\llbracket\{a\},\{1\} \rrbracket \ominus \llbracket\{a\},\{a\} \backslash\{a\} \rrbracket=a \ominus 0=a$.

Theorem 1. Let $E$ be an effect algebra, let $S \subseteq E$. If $S \cup\{1\}$ admits a compatibility support mapping, then $S$ is coexistent.

Proof. Suppose that $S \cup\{1\}$ admits a compatibility support mapping. Let us construct $F_{B}(S)$ as the direct limit of the direct family $\left(2^{2^{A}}: A \in \operatorname{Fin}(S)\right)$, equipped with morphisms of the type $g_{B}^{A}$. After that, we shall define an observable $\alpha: F_{B}(S) \rightarrow E$.

Consider the set

$$
\Gamma_{S}=\bigcup_{A \in \operatorname{Fin}(S)}\left\{(\mathbb{X}, A): \mathbb{X} \subseteq 2^{A}\right\}
$$

and define on it a binary relation $\equiv$ by $(\mathbb{X}, A) \equiv(\mathbb{Y}, B)$ if and only if $g_{A \cup B}^{A}(\mathbb{X})=$ $g_{A \cup B}^{B}(\mathbb{Y})$, that means

$\left\{X \cup C_{A}: X \in \mathbb{X}\right.$ and $\left.C_{A} \subseteq A \cup B \backslash A\right\}=\left\{Y \cup C_{B}: Y \in \mathbb{Y}\right.$ and $\left.C_{B} \subseteq A \cup B \backslash B\right\}$.

Then $F_{B}(S)=\Gamma_{S} / \equiv$ and the operations on $F_{B}(S)$ are defined by

$$
\left.[(\mathbb{X}, A)]_{\equiv \vee} \vee(\mathbb{Y}, B)\right]_{\equiv}=\left[\left(g_{A \cup B}^{A}(\mathbb{X}) \cup g_{A \cup B}^{B}(\mathbb{Y}), A \cup B\right)\right]_{\equiv}
$$

and similarly for the other operations. Then $F_{B}(S)$ is a direct limit of Boolean algebras, hence a Boolean algebra.

Let $\alpha_{S}: F_{B}(S) \rightarrow E$ be a mapping given by the rule $\alpha_{S}\left([(\mathbb{X}, A)]_{\equiv}\right)=\alpha_{A}(\mathbb{X})$. We shall prove that $\alpha_{S}$ is an observable.

Let us prove $\alpha_{S}$ is well-defined. Suppose that $(\mathbb{X}, A) \equiv(\mathbb{Y}, B)$, that means, $g_{A \cup B}^{A}(\mathbb{X})=g_{A \cup B}^{B}(\mathbb{Y})$. By Lemma 4,

$$
\alpha_{A}(\mathbb{X})=\alpha_{A \cup B}\left(g_{A \cup B}^{A}(\mathbb{X})\right)
$$

and

$$
\alpha_{B}(\mathbb{Y})=\alpha_{A \cup B}\left(g_{A \cup B}^{B}(\mathbb{Y})\right),
$$

hence $\alpha_{S}$ is a well-defined mapping.

Let us prove that $\alpha_{S}$ is an observable. The bounds of the Boolean algebra $F_{B}(S)$ are $[(\emptyset, A)]_{\equiv}$ and $\left[\left(2^{A}, A\right)\right]_{\equiv}$, where $A \in \operatorname{Fin}(S)$. Obviously, by Corollary 2 ,

$$
\alpha_{S}\left([(\emptyset, A)]_{\equiv}\right)=\alpha_{A}(\emptyset)=0
$$

and

$$
\alpha_{S}\left(\left[\left(2^{A}, A\right)\right]_{\equiv}\right)=\alpha_{A}\left(2^{A}\right)=1 .
$$


Let $[(\mathbb{X}, A)]_{\equiv}$ and $\left[(\mathbb{Y}, B)_{\equiv}\right]$ be disjoint elements of $F_{B}(S)$, that is, $g_{A \cup B}^{A}(\mathbb{X}) \cap$ $g_{A \cup B}^{B}(\mathbb{Y})=\emptyset$. Then

$$
\begin{array}{r}
\alpha_{S}\left([(\mathbb{X}, A)]_{\equiv} \vee[(\mathbb{Y}, B)]_{\equiv}\right)=\alpha_{S}\left(\left[g_{A \cup B}^{A}(\mathbb{X}) \cup g_{A \cup B}^{B}(\mathbb{Y}), A \cup B\right]_{\equiv}\right)= \\
=\alpha_{A \cup B}\left(g_{A \cup B}^{A}(\mathbb{X}) \cup g_{A \cup B}^{B}(\mathbb{Y})\right) .
\end{array}
$$

Since $\alpha_{A \cup B}$ is an observable,

$$
\alpha_{A \cup B}\left(g_{A \cup B}^{A}(\mathbb{X}) \cup g_{A \cup B}^{B}(\mathbb{Y})\right)=\alpha_{A \cup B}\left(g_{A \cup B}^{A}(\mathbb{X})\right) \oplus \alpha_{A \cup B}\left(g_{A \cup B}^{B}(\mathbb{Y})\right) .
$$

It remains to observe that

$$
\alpha_{A \cup B}\left(g_{A \cup B}^{A}(\mathbb{X})\right)=\alpha_{S}\left([(\mathbb{X}, A)]_{\equiv}\right)
$$

and that

$$
\alpha_{A \cup B}\left(g_{A \cup B}^{B}(\mathbb{Y})\right)=\alpha_{S}\left([(\mathbb{Y}, B)]_{\equiv}\right) .
$$

Let us prove that the range of $\alpha_{S}$ includes $S$. Let $a \in S$. By Corollary 3 , the range of $\alpha_{\{a\}}$ includes $a$ and, by an obvious direct limit argument, the range of $\alpha_{\{a\}}$ is a subset of the range of $\alpha_{S}$.

\section{COMPatibility SUPPORT MAPPINGS FROM OBSERVABLES}

The aim of the single theorem of this section is to prove that every subset $S$ of the range of an observable admits a strong compatibility support mapping.

Theorem 2. For every coexistent subset $S$ of an effect algebra $E, S \cup\{1\}$ admits a strong compatibility support mapping.

Proof. Let $B$ be a Boolean algebra and let $\alpha: B \rightarrow E$ be an observable, let $S$ be a subset of the range of $\alpha$.

For every $a \in S \cup\{1\}$, fix an element $p_{a} \in \alpha^{-1}(a)$ and define

$$
\llbracket U, V \rrbracket=\alpha\left(\left(\bigwedge_{a \in U} p_{a}\right) \wedge\left(\bigvee_{b \in V} p_{b}\right)\right) .
$$

Let us check the condition in the definition of a strong compatibility support mapping. Let $c \notin U, V$. Then

$$
\begin{array}{r}
\llbracket U \cup\{c\},\{1\} \rrbracket \ominus \llbracket U \cup\{c\}, V \rrbracket= \\
=\alpha\left(\left(\bigwedge_{a \in U} p_{a}\right) \wedge p_{c}\right) \ominus \alpha\left(\left(\left(\bigwedge_{a \in U} p_{a}\right) \wedge p_{c}\right) \wedge\left(\bigvee_{b \in V} p_{b}\right)\right) .
\end{array}
$$

To simplify the matters, write

$$
\begin{array}{r}
m_{U}=\left(\bigwedge_{a \in U} p_{a}\right) \\
j_{V}=\left(\bigvee_{b \in V} p_{b}\right)
\end{array}
$$

We can write

$$
\begin{array}{r}
\alpha\left(\left(\bigwedge_{a \in U} p_{a}\right) \wedge p_{c}\right) \ominus \alpha\left(\left(\left(\bigwedge_{a \in U} p_{a}\right) \wedge p_{c}\right) \wedge\left(\bigvee_{b \in V} p_{b}\right)\right)=\alpha\left(m_{U} \wedge p_{c}\right) \ominus \alpha\left(m_{U} \wedge p_{c} \wedge j_{V}\right)= \\
=\alpha\left(\left(m_{U} \wedge p_{c}\right) \ominus\left(m_{U} \wedge p_{c} \wedge j_{V}\right)\right)
\end{array}
$$

Similarly,

$$
\llbracket U, V \cup\{c\} \rrbracket \ominus \llbracket U, V \rrbracket=\alpha\left(\left(m_{U} \wedge\left(p_{c} \vee j_{V}\right)\right) \ominus\left(m_{U} \wedge j_{V}\right)\right) .
$$


Since $B$ is a Boolean algebra,

$$
\left(m_{U} \wedge p_{c}\right) \ominus\left(m_{U} \wedge p_{c} \wedge j_{V}\right)=\left(m_{U} \wedge\left(p_{c} \vee j_{V}\right)\right) \ominus\left(m_{U} \wedge j_{V}\right)
$$

The remaining conditions are trivial to check.

Let us note that, if we start with a non-strong compatibility support mapping, apply Theorem 1 to construct an observable and then apply Theorem 2 to construct a compatibility support mapping, we cannot obtain the compatibility support mapping we started with, since Theorem 2 always produces a strong compatibility support mapping.

\section{Properties of StRong COMPATIBILITy SUPPORT MAPPINGS}

The aim of this section is to prove that several properties of the Example 2 are valid for all strong compatibility support mappings. It remains open whether and which of these properties are valid for all compatibility support mappings.

The main vehicle here is Proposition 2, that is interesting in its own right: it shows that, for a given $S$, every strong compatibility support mapping on $S$ is determined by its $D(.,$.$) .$

Running assumption 2. In this section, we assume the following.

- $E$ is an effect algebra.

- $S$ is a subset of $E$ with $1 \in S$.

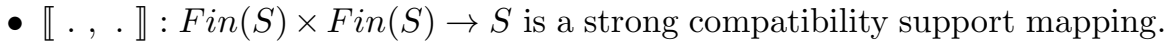

Lemma 5. If $U, V$ are not disjoint, then $\llbracket U, V \rrbracket=\llbracket U,\{1\} \rrbracket$.

Proof. Let $c \in U \cap V$. This implies that $U \cup\{c\}=U$ and $V \cup\{c\}=V$. Therefore, by $\left(\mathrm{e}^{*}\right)$,

$$
\llbracket U,\{1\} \rrbracket \ominus \llbracket U, V \rrbracket=\llbracket U, V \rrbracket \ominus \llbracket U, V \rrbracket=0,
$$

hence $\llbracket U, V \rrbracket=\llbracket U,\{1\} \rrbracket$.

Lemma 6. $\llbracket U \cup\{c\},\{1\} \rrbracket=\llbracket U,\{c\} \rrbracket$.

Proof. Put $V=\emptyset$ in $\left(\mathrm{e}^{*}\right)$ :

$$
\llbracket U \cup\{c\},\{1\} \rrbracket \ominus \llbracket U \cup\{c\}, \emptyset \rrbracket=\llbracket U,\{c\} \rrbracket \ominus \llbracket U, \emptyset \rrbracket .
$$

By condition (c), $\llbracket U \cup\{c\}, \emptyset \rrbracket=\llbracket U, \emptyset \rrbracket=0$, therefore

$$
\llbracket U \cup\{c\},\{1\} \rrbracket=\llbracket U,\{c\} \rrbracket .
$$

Proposition 2. Let $U, V \subseteq S$.

(1) If $U \cap V \neq \emptyset$, then $\llbracket U, V \rrbracket=\llbracket U,\{1\} \rrbracket=D(U, U)$.

(2) If $U \cap V=\emptyset$, then

$$
\llbracket U, V \rrbracket=\bigoplus_{\emptyset \neq Y \subseteq V} D(U \cup Y, U \cup V) .
$$

Proof.

(1) By Proposition $\square \llbracket \llbracket U, V \rrbracket=\llbracket U,\{1\} \rrbracket$ and

$$
D(U, U)=\llbracket U,\{1\} \rrbracket \ominus \llbracket U, \emptyset \rrbracket=\llbracket U,\{1\} \rrbracket \ominus 0=\llbracket U,\{1\} \rrbracket .
$$


(2) By Lemma 3 ,

$$
D(U, U)=\bigoplus_{Y \subseteq V} D(U \cup Y, U \cup V)
$$

Therefore,

$$
D(U, U) \ominus D(U, U \cup V)=\bigoplus_{\emptyset \neq Y \subseteq V} D(U \cup Y, U \cup V) .
$$

Moreover,

$$
\begin{array}{r}
D(U, U) \ominus D(U, U \cup V)=(\llbracket U,\{1\} \rrbracket \ominus \llbracket U, \emptyset \rrbracket) \ominus(\llbracket U,\{1\} \rrbracket \ominus \llbracket U, V \rrbracket)= \\
=\llbracket U, V \rrbracket \ominus \llbracket U, \emptyset \rrbracket=\llbracket U, V \rrbracket \ominus 0=\llbracket U, V \rrbracket .
\end{array}
$$

Proposition 3. If $U_{1} \subseteq U_{2}$, then $\llbracket U_{1}, V \rrbracket \geq \llbracket U_{2}, V \rrbracket$.

Proof.

(Case 1) Suppose that $U_{1} \cap V \neq \emptyset$. Then $U_{2} \cap V \neq \emptyset$. By Proposition 2 and Lemma 3

$$
\llbracket U_{1}, V \rrbracket=D\left(U_{1}, U_{1}\right)=\bigoplus_{Y \subseteq U_{2} \backslash U_{1}} D\left(U_{1} \cup Y, U_{2}\right) \geq D\left(U_{2}, U_{2}\right)=\llbracket U_{2}, V \rrbracket .
$$

(Case 2) Suppose that $U_{2} \cap V=\emptyset$. Then $U_{1} \cap V=\emptyset$. By Proposition 2 ,

$$
\llbracket U_{1}, V \rrbracket=\bigoplus_{\emptyset \neq Y \subseteq V} D\left(U_{1} \cup Y, U_{1} \cup V\right) .
$$

By Lemma 3, for every $\emptyset \neq Y \subseteq V$,

$$
D\left(U_{1} \cup Y, U_{1} \cup V\right)=\bigoplus_{W \subseteq U_{2} \backslash U_{1}} D\left(U_{1} \cup Y \cup W, U_{2} \cup V\right) .
$$

Obviously (put $W=U_{2} \backslash U_{1}$ ), this implies that

$$
D\left(U_{1} \cup Y, U_{1} \cup V\right) \geq D\left(U_{2} \cup Y, U_{2} \cup V\right),
$$

hence we may write

$$
\llbracket U_{1}, V \rrbracket=\bigoplus_{\emptyset \neq Y \subseteq V} D\left(U_{1} \cup Y, U_{1} \cup V\right) \geq \bigoplus_{\emptyset \neq Y \subseteq V} D\left(U_{2} \cup Y, U_{2} \cup V\right) .
$$

It remains to apply Proposition 2 again:

$$
\bigoplus_{\emptyset \neq Y \subseteq V} D\left(U_{2} \cup Y, U_{2} \cup V\right)=\llbracket U_{2}, V \rrbracket .
$$

(Case 3) Suppose that $U_{1} \cap V=\emptyset$ and $U_{2} \cap V \neq \emptyset$. By Proposition 2 ,

$$
\llbracket U_{1}, V \rrbracket=\bigoplus_{\emptyset \neq Y \subseteq V} D\left(U_{1} \cup Y, U_{1} \cup V\right) .
$$

By Lemma 3,

$$
D\left(U_{1} \cup Y, U_{1} \cup V\right)=\bigoplus_{W \subseteq U_{2} \backslash\left(U_{1} \cup V\right)} D\left(U_{1} \cup W \cup Y, U_{2} \cup V\right)
$$

We can put $W=U_{2} \backslash\left(U_{1} \cup V\right)$, proving that

$$
D\left(U_{1} \cup Y, U_{1} \cup V\right) \geq D\left(\left(U_{2} \backslash V\right) \cup Y, U_{2} \cup V\right) .
$$


Therefore,

$$
\begin{aligned}
& \llbracket U_{1}, V \rrbracket=\bigoplus_{\emptyset \neq Y \subseteq V} D\left(U_{1} \cup Y, U_{1} \cup V\right) \geq \bigoplus_{\emptyset \neq Y \subseteq V} D\left(\left(U_{2} \backslash V\right) \cup Y, U_{2} \cup V\right) \geq \\
& \bigoplus_{V \cap U_{2} \subseteq Y \subseteq V} D\left(\left(U_{2} \backslash V\right) \cup Y, U_{2} \cup V\right) .
\end{aligned}
$$

For every $V \cap U_{2} \subseteq Y \subseteq V$, there is exactly one $Z \subseteq V \backslash U_{2}$ such that

$$
\left(U_{2} \backslash V\right) \cup Y=U_{2} \cup Z \text {. }
$$

Thus, we can rewrite

$$
\bigoplus_{V \cap U_{2} \subseteq Y \subseteq V} D\left(\left(U_{2} \backslash V\right) \cup Y, U_{2} \cup V\right)=\bigoplus_{Z \subseteq V \backslash U_{2}} D\left(U_{2} \cup Z, U_{2} \cup V\right) .
$$

By Lemma 3 and Proposition 2 ,

$$
\bigoplus_{Z \subseteq V \backslash U_{2}} D\left(U_{2} \cup Z, U_{2} \cup V\right)=D\left(U_{2}, U_{2}\right)=\llbracket U_{2}, V \rrbracket .
$$

Proposition 4. $\llbracket U,\{1\} \rrbracket$ is a lower bound of $U$.

Proof. Any element is a lower bound of $\emptyset$.

Suppose that the proposition is true for some $U$ and pick $c \in S \backslash U$. By Proposition 3 ,

$$
\llbracket U \cup\{c\},\{1\} \rrbracket \leq \llbracket U,\{1\} \rrbracket .
$$

By the induction hypothesis, $\llbracket U,\{1\} \rrbracket$ is a lower bound of $U$. It remains to prove that $\llbracket U \cup\{c\},\{1\} \rrbracket \leq c$. By Proposition 6 , $\llbracket U \cup\{c\},\{1\} \rrbracket=\llbracket U,\{c\} \rrbracket$. By Proposition 3 and condition $(\mathrm{d}), \llbracket U,\{c\} \rrbracket \leq \llbracket \emptyset,\{c\} \rrbracket=c$.

Corollary 4. $\llbracket U, V \rrbracket$ is a lower bound of $U$.

Proof. By Proposition $\llbracket$, $U,\{1\} \rrbracket$ is a lower bound of $U$. By condition (b), $\llbracket U, V \rrbracket \leq$ $\llbracket U,\{1\} \rrbracket$.

Proposition 5. $\llbracket \emptyset, V \rrbracket$ is an upper bound of $V$.

Proof. Any element is an upper bound of $\emptyset$. (a),

Suppose that the proposition is true for some $V$ and pick $c \in S \backslash V$. By condition

$$
\llbracket \emptyset, V \rrbracket \leq \llbracket \emptyset, V \cup\{c\} \rrbracket
$$

and by induction hypothesis, $\llbracket \emptyset, V \rrbracket$ is an upper bound of $V$. It remains to prove that $c \leq \llbracket \emptyset, V \cup\{c\} \rrbracket$.

Put $U=\emptyset$ in condition $\left(\mathrm{e}^{*}\right)$ :

$$
\llbracket\{c\},\{1\} \rrbracket \ominus \llbracket\{c\}, V \rrbracket=\llbracket \emptyset, V \cup\{c\} \rrbracket \ominus \llbracket \emptyset, V \rrbracket .
$$

Add $\llbracket \emptyset, V \rrbracket$ to both sides to obtain

$$
(\llbracket\{c\},\{1\} \rrbracket \ominus \llbracket\{c\}, V \rrbracket) \oplus \llbracket \emptyset, V \rrbracket=\llbracket \emptyset, V \cup\{c\} \rrbracket .
$$

As $\llbracket\{c\}, V \rrbracket \leq \llbracket \emptyset, V \rrbracket$,

$$
\llbracket\{c\},\{1\} \rrbracket \leq(\llbracket\{c\},\{1\} \rrbracket \ominus \llbracket\{c\}, V \rrbracket) \oplus \llbracket \emptyset, V \rrbracket .
$$

By Lemma 1, $\llbracket\{c\},\{1\} \rrbracket=c$. 


\section{COMPATIBILITY SUPPORT MAPPINGS AND WITNESS MAPPINGS}

Let $(G, \leq)$ be a partially ordered abelian group and $u \in G$ be a positive element. For $0 \leq a, b \leq u$, define $a \oplus b$ if and only if $a+b \leq u$ and put $a \oplus b=a+b$. With such a partial operation $\oplus$, the closed interval

$$
[0, u]_{G}=\{x \in G: 0 \leq x \leq u\}
$$

becomes an effect algebra $\left([0, u]_{G}, \oplus, 0, u\right)$. Effect algebras which arise from partially ordered abelian groups in this way are called interval effect algebras, see 2].

Let $E$ be an interval effect algebra in a partially ordered abelian group $G$. Let $S \subseteq E$. Let us write $\operatorname{Fin}(S)$ for the set of all finite subsets of $S$. We write $I(F i n(S))$ for the set of all comparable elements of the poset $(\operatorname{Fin}(S), \subseteq)$, that means,

$$
I(\operatorname{Fin}(S))=\{(X, Y) \in \operatorname{Fin}(S) \times \operatorname{Fin}(S): X \subseteq Y\} .
$$

For every mapping $\beta: \operatorname{Fin}(S) \rightarrow G$, we define a mapping $D_{\beta}: I(\operatorname{Fin}(S)) \rightarrow G$. For $(X, A) \in I(\operatorname{Fin}(S))$, the value $D_{\beta}(X, A) \in G$ is given by the rule

$$
D_{\beta}(X, A):=\sum_{X \subseteq Z \subseteq A}(-1)^{|X|+|Z|} \beta(Z) .
$$

In [7, we introduced and studied the following notion:

Definition 2. Let $E$ be an interval effect algebra. We say that a mapping $\beta$ : $\operatorname{Fin}(S) \rightarrow E$ is a witness mapping for $S$ if and only if the following conditions are satisfied.

(A1) $\beta(\emptyset)=1$,

(A2) for all $c \in S, \beta(\{c\})=c$,

(A3) for all $(X, A) \in I(F i n(S)), D_{\beta}(X, A) \geq 0$.

We proved there, that a subset $S$ of an interval effect algebra $E$ is coexistent if and only if there is a witness $06 \beta: \operatorname{Fin}(S) \rightarrow E$.

The aim of this section is to explore the connection between the notion of a witness mapping and the notion of compatibility support mappings.

Proposition 6. Let $E$ be an interval effect algebra, let $S$ be a subset of $E$ with $1 \in$ S. Suppose there is a compatibility support mapping $\llbracket ., . \rrbracket: F i n(S) \times F i n(S) \rightarrow$ $S$. Then $\beta: F i n(S) \rightarrow E$, given by $\beta(X)=\llbracket X,\{1\} \rrbracket$ is a witness mapping and $D(X, A)=D_{\beta}(X, A)$, for all $(X, A) \in I(F i n(S))$.

Proof. We see that, by the condition (d) of Definition 1,

$$
\beta(\emptyset)=\llbracket \emptyset,\{1\} \rrbracket=1,
$$

so the condition (A1) of Definition 2 is satisfied. By Lemma 1.

$$
\beta(\{c\})=\llbracket\{c\},\{1\} \rrbracket=c,
$$

hence (A2) is satisfied.

For the proof of (A3), it suffices to prove that $D(X, A)=D_{\beta}(X, A)$, for all $(X, A) \in I(\operatorname{Fin}(S))$. The positivity of $D_{\beta}$ then follows from the positivity of $D$. The proof goes by induction with respect to $|A \backslash X|$.

If $|A \backslash X|=0$, then $A=X$ and

$$
D_{\beta}(X, A)=\beta(X)=\llbracket X,\{1\} \rrbracket=\llbracket X,\{1\} \rrbracket \ominus 0=\llbracket X,\{1\} \rrbracket \ominus \llbracket X, \emptyset \rrbracket=D(X, A) .
$$


Suppose that $D(X, A)=D_{\beta}(X, A)$, for all $(X, A) \in I(F i n(S))$ such that $\mid A \backslash$ $X \mid=n$. Let $(Y, B) \in I(F i n(S))$ be such that $|B \backslash Y|=n+1$. Pick $c \in B \backslash Y$ and put $X=Y, A=B \backslash\{c\}$.

By Lemma 1 of [7, for any mapping $\beta: \operatorname{Fin}(S) \rightarrow E$, for all $(X, A) \in I(\operatorname{Fin}(S))$ and for all $c \in S \backslash A$, the following equality is satisfied:

$$
D_{\beta}(X, A)=D_{\beta}(X, A \cup\{c\})+D_{\beta}(X \cup\{c\}, A \cup\{c\}) .
$$

Therefore,

$$
D_{\beta}(Y, B)=D_{\beta}(X, A \cup\{c\})=D_{\beta}(X, A) \ominus D_{\beta}(X \cup\{c\}, A \cup\{c\}) .
$$

By the induction hypothesis, $D_{\beta}(X, A)=D(X, A)$ and $D_{\beta}(X \cup\{c\}, A \cup\{c\})=$ $D_{\beta}(X \cup\{c\}, A \cup\{c\})$. Thus,

$$
D_{\beta}(Y, B)=D(X, A) \ominus D(X \cup\{c\}, A \cup\{c\}) .
$$

By Lemma 2,

$$
D(X, A) \ominus D(X \cup\{c\}, A \cup\{c\})=D(X, A \cup\{c\})=D_{\beta}(Y, B) .
$$

The following problem remains open.

Problem 1. Let $E$ be an effect algebra, let $S \subseteq E$, let $\beta: \operatorname{Fin}(S) \rightarrow E$ be a witness mapping. Is there always a compatibility support mapping $\llbracket ., . \rrbracket$ : $\operatorname{Fin}(S) \times \operatorname{Fin}(S) \rightarrow S$ such that $\beta(X)=\llbracket X,\{1\} \rrbracket$ ?

\section{REFERENCES}

[1] M.K. Bennett and D.J. Foulis. Phi-symmetric effect algebras. Foundations of Physics, 25:16999722, 1995.

[2] M.K. Bennett and D.J. Foulis. Interval and scale effect algebras. Advances in Applied Mathematics, 19:200-215, 1997.

[3] G. Cattaneo, M. L. Dalla Chiara, R. Giuntini, and S. Pulmannová. Effect Algebras and ParaBoolean Manifolds. International Journal of Theoretical Physics, 39(3):551-564, 2000.

[4] F. Chovanec and F. Kôpka. Boolean D-posets. Tatra Mt. Math. Publ, 10:183-197, 1997.

[5] D.J. Foulis and M.K. Bennett. Effect algebras and unsharp quantum logics. Found. Phys., 24:1325-1346, 1994

[6] R. Giuntini and H. Greuling. Toward a formal language for unsharp properties. Found. Phys., 19:931-945, 1989.

[7] G. Jenča. Coexistence in interval effect algebras. 2009, arXiv:quant-ph/0910.2823.

[8] F. Kôpka and F. Chovanec. D-posets. Math. Slovaca, 44:21-34, 1994.

[9] S. Pulmannová. A note on observables on MV-algebras. Soft Computing, 4(1):45-48, 2000.

Department of Mathematics and Descriptive Geometry, Faculty of Civil Engineering, Radlinského 11, Bratislava 813 68, Slovak Republic

E-mail address: gejza.jenca@stuba.sk 